\title{
Three-dimensional greyscale transrectal ultrasound-guidance and biopsy core preembedding for detection of prostate cancer: Dutch clinical cohort study
}

Anouk Anna Maria Arnoldus van der $\mathrm{Aa}^{1,4^{*}+}$, Christophe Koenraad Mannaerts ${ }^{5+}$, Maudy C. W. Gayet ${ }^{1,4}$, Johannes Cornelis van der Linden², Barthold Philip Schrier ${ }^{1}$, J. P. Michiel Sedelaar ${ }^{3}$, Massimo Mischi', Harrie P. Beerlage ${ }^{4,5}$ and Hessel Wijkstra ${ }^{4,5}$

\begin{abstract}
Background: To overcome the limitations regarding two dimensional (2D) greyscale (GS) transrectal ultrasound (TRUS)guided biopsy in prostate cancer (PCa) detection and tissue packaging in biopsy processing, there is an ongoing focus on new imaging and pathology techniques. A three-dimensional (3D) model of the prostate with biopsy needle guidance can be generate by the Navigo ${ }^{T M}$ workstation (UC-care, Israel). The SmartBX ${ }^{\mathrm{TM}}$ system (UC-care, Israel) provides a prostate biopsy core preembedding method. The aim of this study was to compare cancer detection rates between the 3D TRUSguidance and preembedding method with conventional 2D GS TRUS-guidance among patients undergoing prostate biopsies.

Methods: We retrospectively analyzed the records of all patients who underwent prostate biopsies for PCa detection at our institution from 2007 to 2016. The cohort was divided into a 2D GS TRUS-guidance cohort (from 2007 to 2013, $n=1149$ ) and a 3D GS TRUS-guidance with preembedding cohort (from 2013 to 2016, $n=469$ ). Effect of 3D GS TRUS-guidance with preembedding on detection rate of PCa and clinically significant PCa (Gleason score $\geq 7$ or $>2$ biopsy cores with a Gleason score 6) was compared to 2D GS TRUS-guidance using regression models.

Results: Detection rate of PCa and clinically significant PCa was 39.0 and $24.9 \%$ in the 3D GS TRUS cohort compared to 33.5 and $19.0 \%$ in the 2D GS TRUS cohort, respectively. On multivariate regression analysis the use of 3D GS TRUS-guidance with preembedding was associated with a significant increase in detection rate of PCa (aOR $=1.33 ; 95 \% \mathrm{Cl}: 1.03-1.72)$ and clinically significant PCa $(a O R=1.47 ; 95 \% \mathrm{Cl}: 1.09-1.98)$.

Conclusion: Our results suggest that 3D GS TRUS-guidance with biopsy core preembedding improves PCa and clinically significant PCa detection compared to 2D GS TRUS-guidance. Additional studies are needed to justify the application of these systems in clinical practice.
\end{abstract}

Keywords: Prostatic neoplasms, Biopsy, Three-dimensional imaging, Tissue embedding, Diagnosis

\footnotetext{
*Correspondence: A.v.d.Aa@jbz.nl

${ }^{+}$Anouk Anna Maria Arnoldus van der Aa and Christophe Koenraad

Mannaerts contributed equally to this work.

'Department of Urology, Jeroen Bosch Hospital, Post office box 90153,

's-Hertogenbosch 5200 ME, The Netherlands

${ }^{4}$ Electrical Engineering, Eindhoven University of Technology, Eindhoven, The

Netherlands

Full list of author information is available at the end of the article
}

(c) The Author(s). 2019 Open Access This article is distributed under the terms of the Creative Commons Attribution 4.0 International License (http://creativecommons.org/licenses/by/4.0/), which permits unrestricted use, distribution, and reproduction in any medium, provided you give appropriate credit to the original author(s) and the source, provide a link to the Creative Commons license, and indicate if changes were made. The Creative Commons Public Domain Dedication waiver (http://creativecommons.org/publicdomain/zero/1.0/) applies to the data made available in this article, unless otherwise stated. 


\section{Background}

Prostate cancer $(\mathrm{PCa})$ is the most common malignancy among Western males [1]. Early detection of PCa reduces PCa-related mortality and reduces the risk of being diagnosed and developing advanced or metastatic disease [2].

PCa detection with systematic prostate biopsies under ultrasound guidance, in men identified with elevated serum prostate-specific antigen (PSA) and / or abnormal digital rectal examination (DRE), is considered as the preferred diagnostic method by guidelines from leading organizations [3, 4]. Although standard two dimensional (2D) greyscale (GS) transrectal ultrasound (TRUS)-guided biopsy is able to detect $\mathrm{PCa}$, it has well known limitations. 2D GS TRUS has low sensitivity and specificity for detection of $\mathrm{PCa}$ and is therefore used to guide prostate biopsies rather than used as a diagnostic modality. Even in TRUS-guided biopsy there is still a $20-30 \%$ risk of sampling error with underdiagnosis of $\mathrm{PCa}$ that requires definitive treatment as a result $[5,6]$. There is also poor correlation with final pathology [7-9]. Unfortunately, imaging is not the only part with influence on $\mathrm{PCa}$ detection as inadequate tissue packaging in biopsy processing also tends to reduce PCa detection outcomes [10].

To overcome these limitations and improve PCa detection, there is an ongoing focus on new imaging and pathology techniques.

3D GS TRUS is an imaging method that allows simultaneous imaging of the prostate in axial and sagittal plane, followed by a computer reconstructed third coronal plane as well as a 3D image [5]. Effectiveness of 3D GS TRUS in detection and local staging of PCa is controversial as limited studies reported different outcomes $[5,11-13]$. However, the feasibility of 3D TRUS in assisting biopsy needle guidance was recognised in phantom and patient studies [14-16]. More recently, Peltier et al. reported significant higher detection rates of clinically significant PCa using a 3D TRUS-guided biopsy system in clinical practice [17].

Biopsy processing with preembedding methods of prostate biopsy cores result in optimal tissue presentation with preservation of shape and avoidance of artefacts and deformation, and have shown to improve histologic yield [18, 19]. Moreover, Rogatsch et al. demonstrated that the improvement of histologic yield using an optimized preembedding method led to a higher frequency of PCa diagnosis [20].

Based on these findings, prostate biopsy sessions in our institution are performed since September 2013 with a phantom validated 3D GS TRUS-guided biopsy system $\left(\mathrm{Navigo}^{\mathrm{Tm}}\right.$ workstation, UC-care Medical Systems Israel) and a semi-automated prostate biopsy core preembedding method proven to improve histologic yield (the SmartBX $^{\mathrm{Tm}}$ system, UC-care Medical Systems Israel) [15, 21]. The purpose of the present study was to investigate the value of 3D GS TRUS-guidance and biopsy core preembedding in the detection of (clinically significant) $\mathrm{PCa}$ compared to conventional 2D GS TRUS-guidance in a consecutive cohort of men undergoing systematic prostate biopsies due to a clinical suspicion of PCa.

\section{Methods}

In this retrospective cohort study, all consecutive men who underwent prostate biopsies between January 2007 and January 2016 in the Urology Department at the Jeroen Bosch Hospital in 's Hertogenbosch, the Netherlands, were included. Patient files were consulted for relevant information. Prostate biopsy sessions performed in patients with no prior history of PCa and a serum PSA level between 4.0 and $10.0 \mu \mathrm{g} / \mathrm{L}$ or PSA $<4.0 \mu \mathrm{g} / \mathrm{L}$ and a suspicious DRE were included in the study. We divided the eligible prostate biopsy sessions into two cohorts. From January 2007 up to September 2013 consecutive biopsy sessions were performed in the 2D TRUS cohort. The 3D TRUS cohort consisted of the consecutive biopsy sessions with preembedding from September 2013 up to January 2016.

\section{D TRUS cohort protocol}

All prostate biopsy sessions were performed with prophylactic antibiotics in the lateral decubitus position. The prostate gland was assessed and scanned and prostate volume measurements were done using the ellipsoid formula, using a BK medical ultrasound machine (type 2202) and BK medical sidefire probe (type 8808 , 6-10 MHz). A 12-core biopsy protocol, under periprostatic local anaesthesia, was then performed consisting of biopsies on both sides in the peripheral zone, 2 biopsies of each base, mid and apex. The biopsy was performed using a spring-loaded gun with 18-gauge needles (Bard Magnum biopsy gun). Biopsy cores were placed in two vials, each vial containing 6 cores of one prostate lobe. Prostate cores were processed and examined in the hospital pathology laboratory. Number of cores per vial, length of biopsy cores, number of positive biopsy cores, ISUP 2005 Gleason score and tumour volume\% of biopsy cores (since 2012) were reported.

\section{D TRUS cohort protocol}

The 3D GS TRUS protocol is basically similar to the 2D TRUS protocol. The 3D GS TRUS system (Navigo $^{\text {тм }}$ workstation) was incorporated side by side in the room with $2 \mathrm{D}$ ultrasound images transferred and displayed on the 3D TRUS screen. A 3D model of the prostate was built after prostate volume measurements and planimetry was done. The outline of the prostate border was manually traced and recorded on a slice-by-slice basis. In real time using an 
electromagnetic system (Fig. 1), the 12-core biopsy protocol was performed with tracking, displaying and recording of biopsy needle trajectory locations. Previously marked regions of interest were shown with colour indications on screen and sampled. Biopsy cores were fixed separately on six different cassettes (two cores of apex, mid and base on both sides) using the Smart-Bx device (Additional file 2). SmartBx ${ }^{\text {tim }}$ device is a semi-automated prostate biopsy core preembedding method, with a designed membrane to which the cores stay attached throughout pathology lab processing, and placed in two formalin-filled vials (3 cassettes in each vial with two cores of apex, mid and base of one prostate lobe).

In both cohorts, biopsy sessions were performed by different operators. Operator was recorded for analysis and split into experienced ( $>100$ procedures/year and $>$ 10 years of experience using TRUS guided biopsies) and less experienced ( $<100$ procedures/year). The following primary outcomes were compared: detection rate of $\mathrm{PCa}$ and detection rate of clinically significant (Gleason score $\geq 7$ or Gleason score 6 with at least 3 positive biopsy cores). The following secondary outcomes were compared in patients with $\mathrm{PCa}$ at biopsy: amount of positive biopsy cores; ratio positive biopsy cores per total biopsy cores; tumour volume\% of biopsy cores and Gleason score.

\section{Statistical}

Statistical analyses were performed using SPSS Statistics $23.0^{\circ}$. Descriptive statistics were used to summarize baseline characteristics. Continuous variables were presented with means and standard deviations (SD) or median and interquartile ranges (IQR) based on symmetrical distribution with the independent two sample t-test or Mann-Whitney $U$ test used to assess differences, respectively. Percentage scores were presented for categorical variables with differences assessed using the Pearson Chi-Square test. To determine association between (clinically significant) PCa and the 3D GS TRUS-guided system with preembedding we calculated the crude and adjusted odds ratio with their $95 \%$ confidence intervals $(\mathrm{CI})$ for each outcome compared to the 2D GS TRUS cohort using univariate and multivariate logistic regression analyses. Covariables were entered on multivariate analysis, selected based on literature and/or performance: inclusion in the adjusted model when addition of the variable resulted in at least $10 \%$ change in the regression coefficient. A $P<0.05$ was considered statistically significant in all analyses [3, 22-24].

\section{Results}

Between January 2007 and January 2016 we performed 2917 prostate biopsy sessions in 2171 different patients (Fig. 2). 2D TRUS biopsies were performed from 2007 to 2013 and 3D TRUS biopsies were performed from 2013

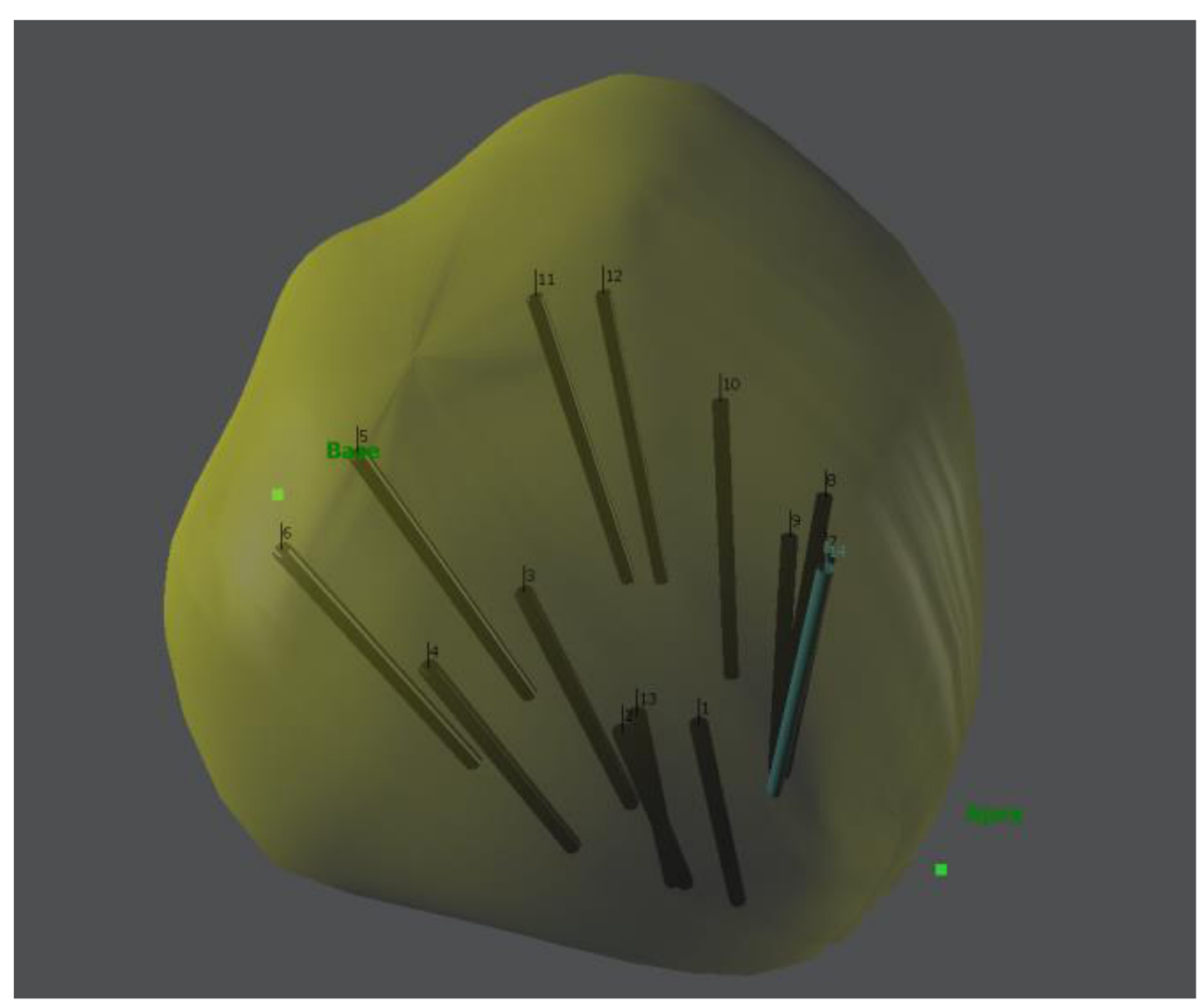

Fig. $1 \mathrm{Navigo}^{\mathrm{TM}}$ 3D model with biopsy locations 


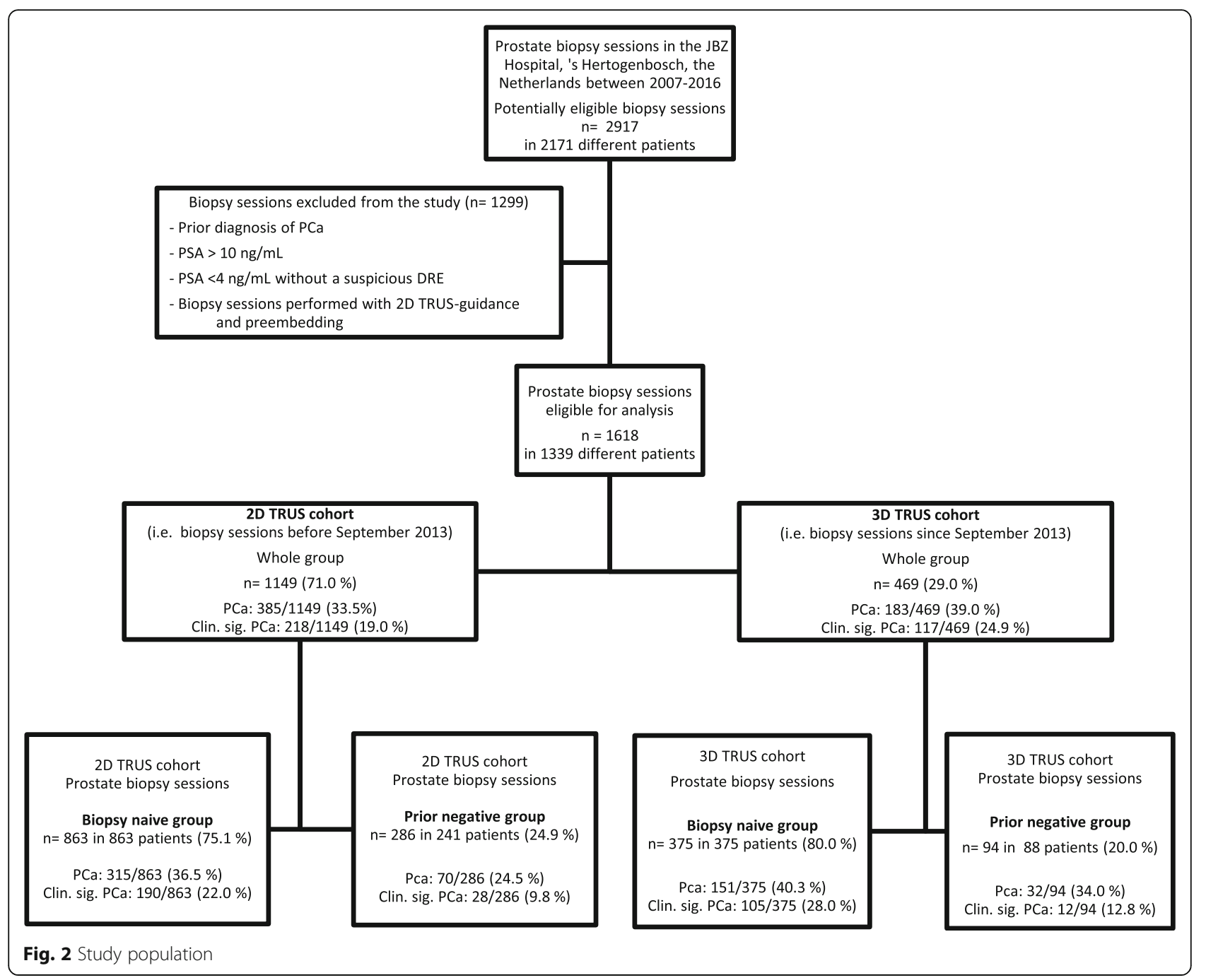

to 2016. A total of 1618 prostate biopsy sessions in 1339 different patients met inclusion criteria. 469 (29.0\%) and $1149(71.0 \%)$ biopsy sessions were performed in the 3D TRUS and 2D TRUS cohort respectively. Patients with missing data on clinical, biopsy and pathology variables (PSA $n=4$, DRE $n=112$, prostate volume $n=13$, length of cores $n=20$ ) were excluded for multivariate analyses. Additionally, information on primary outcome was inadequate for 45 biopsy sessions and therefore these sessions were revised by one uropathologist.

\section{Patient and biopsy characteristics}

Several characteristics differed among the two cohorts for whole group and subgroups (biopsy naive and prior negative biopsy sessions only) analyses (Table 1). For whole group analysis, prostate biopsy sessions in the 3D TRUS cohort were more likely done in older patients (mean age in years; 64.6 vs. $63.0, p<0.001$ ), in patients with larger prostate volumes (median volume in cc; 46 vs. $40, p$ $<0.001)$, in a biopsy naive setting $(80.0 \%$ vs. $75.1 \%$, $p<0.05)$ and by the experienced operator $(73.6 \%$ vs. $43.3 \%, p<0.001)$ compared to the 2D TRUS cohort. The amount of biopsy cores taken per session was comparable in both cohorts (mean cores 12.3 vs. $12.3, p=0.555$ ), while fragmentation of biopsy cores occurred significantly less often in the 3D TRUS cohort (37.7\% vs. $1.9 \%, p<0.001)$. The length of biopsy cores in millimetres was significantly larger in the 3D TRUS cohort (mean length in $\mathrm{mm} ; 16.08$ vs. $12.38, p<0.001)$.

\section{Detection rate}

In the 3D TRUS cohort, a total of 183 out of 469 (39.0\%) and 117 out of 469 (24.9\%) were found positive for PCa and clinically significant PCa compared to 335 out of 1149 (33.5\%) and 218 out of 1149 (19.0\%) in the 2D TRUS cohort, respectively (Table 2). On univariate analysis the 3D TRUS cohort was significantly associated with detection of $\mathrm{PCa}(\mathrm{OR}=1.27,95 \%$ CI $1.02-1.59)$ and clinically significant PCa (OR 1.42, 95\% CI 1.101.83). On multivariate regression analysis with age, DRE, 
Table 1 Patient and biopsy characteristics

\begin{tabular}{|c|c|c|c|c|}
\hline & Total & 2D TRUS & 3D TRUS & $P$ value \\
\hline \multicolumn{5}{|l|}{ Whole group (1339 patients) } \\
\hline Number of biopsy sessions & $N=1618$ & $N=1149(71.0 \%)$ & $N=469(29.0 \%)$ & \\
\hline Age in years, mean (SD) & 63.4 (SD: 6.30) & 63.0 (SD: 6.27) & 64.6 (SD: 6.24) & $<0.001$ \\
\hline PSA in ng/mL, mean (SD) & 6.92 (SD: 1.84) & 6.95 (SD: 1.85) & 6.84 (SD: 1.84) & 0.248 \\
\hline \multicolumn{5}{|l|}{ DRE: } \\
\hline - benign, n (\%) & $1028(68.3 \%)$ & 739 (69.3\%) & $289(65.7 \%)$ & \multirow[t]{2}{*}{0.167} \\
\hline • suspicious, n (\%) & $478(31.7 \%)$ & $327(30.7 \%)$ & $151(34.3 \%)$ & \\
\hline Prostate volume in $\mathrm{ml}$, median (IQR) & $41(30-56)$ & $40(30-54)$ & $46(33-63)$ & $<0.001$ \\
\hline \multicolumn{5}{|l|}{ Type of biopsy sessions: } \\
\hline • Biopsy naive, n (\%) & $1238(76.5 \%)$ & $863(75.1 \%)$ & $375(80.0 \%)$ & \multirow[t]{2}{*}{0.037} \\
\hline - Prior negative, n (\%) & $380(23.5 \%)$ & $286(24.9 \%)$ & $94(20.0 \%)$ & \\
\hline \multicolumn{5}{|l|}{ Type of operator: } \\
\hline • Experienced, n (\%) & $843(52.1 \%)$ & 498 (43.3\%) & $345(73.6 \%)$ & \multirow[t]{2}{*}{$<0.001$} \\
\hline - Less experienced, n (\%) & 775 (47.9\%) & $651(56.7 \%)$ & $124(26.4 \%)$ & \\
\hline Biopsy cores per session, mean (SD) & 12.3 (SD 1.04) & 12.3 (SD 1.05) & 12.3 (SD 1.02) & 0.555 \\
\hline Biopsy sessions with fragmentation, n (\%) & 442 (27.3\%) & $433(37.7 \%)$ & $9(1.9 \%)$ & $<0.001$ \\
\hline Length of biopsy cores in $\mathrm{mm}$, mean (SD) ${ }^{\mathrm{a}}$ & 13.84 (SD: 2.98) & 12.38 (SD: 2.82) & 16.08 (SD: 1.41) & $<0.001$ \\
\hline \multicolumn{5}{|l|}{ Biopsy naïve group (1238 patients) } \\
\hline Number of biopsy sessions & $N=1238$ & $N=863(69.7 \%)$ & $N=375(30.3 \%)$ & \\
\hline Age in years, mean (SD) & 63.5 (SD: 6.46) & 63.0 (SD: 6.43) & 64.7 (SD: 6.35) & $<0.001$ \\
\hline PSA in ng/mL, mean (SD) & 6.73 (SD: 1.87) & 6.72 (SD: 1.87) & 6.74 (SD: 1.86) & 0.877 \\
\hline \multicolumn{5}{|l|}{ DRE: } \\
\hline • benign, n (\%) & 777 (66.9\%) & $556(68.6 \%)$ & $221(63.0 \%)$ & \multirow[t]{2}{*}{0.063} \\
\hline • suspicious, n (\%) & 385 (33.1\%) & $255(31.4 \%)$ & $130(37.0 \%)$ & \\
\hline Prostate volume in ml, median (IQR) & $40(30-55)$ & $40(29-52)$ & $44(32-60)$ & $<0.001$ \\
\hline \multicolumn{5}{|l|}{ Type of operator: } \\
\hline - Experienced, n (\%) & $643(51.9 \%)$ & $368(42.6 \%)$ & $275(73.3 \%)$ & \multirow[t]{2}{*}{$<0.001$} \\
\hline • Less experienced, n (\%) & $595(48.1 \%)$ & $495(57.4 \%)$ & $100(26.7 \%)$ & \\
\hline Biopsy cores per session, mean (SD) & 12.2 (SD 0.79) & $12.2(\mathrm{SD} 0.80)$ & $12.2(\mathrm{SD} 0.77)$ & 0.779 \\
\hline Biopsy sessions with fragmentation, n (\%) & $334(27.0 \%)$ & $325(37.7 \%)$ & $9(2.4 \%)$ & $<0.001$ \\
\hline Length of biopsy cores in mm, mean (SD) ${ }^{a}$ & 13.84 (SD: 2.96) & 12.33 (SD: 2.78) & 16.07 (SD: 1.40) & $<0.001$ \\
\hline \multicolumn{5}{|l|}{ Prior negative group (321 patients) } \\
\hline Number of biopsy sessions & $N=380$ & $N=286(75.3 \%)$ & $N=94(24.7 \%)$ & \\
\hline \multicolumn{5}{|l|}{ Prior negative sessions specified: } \\
\hline - Second session & $300(78.9 \%)$ & $225(78.7 \%)$ & $75(79.8 \%)$ & \multirow[t]{4}{*}{0.658} \\
\hline - Third session & $64(16.8 \%)$ & $47(16.4 \%)$ & $17(18.1 \%)$ & \\
\hline - Fourth session & $14(3.7 \%)$ & $12(4.2 \%)$ & $2(2.1 \%)$ & \\
\hline - Fifth session & $2(0.5 \%)$ & $2(0.7 \%)$ & 0 & \\
\hline Age in years, mean (SD) & 63.1 (SD: 5.79) & 62.8 (SD: 5.80) & 63.9 (SD: 5.73) & 0.135 \\
\hline PSA in ng/mL, mean (SD) & 7.54 (SD: 1.60) & 7.65 (SD: 1.54) & 7.20 (SD: 1.73) & 0.023 \\
\hline \multicolumn{5}{|l|}{ DRE: } \\
\hline • benign, n (\%) & $251(73.0 \%)$ & $183(71.8 \%)$ & $68(76.4 \%)$ & \multirow[t]{2}{*}{0.396} \\
\hline • suspicious, n (\%) & $93(27.0 \%)$ & $72(28.2 \%)$ & $21(23.6 \%)$ & \\
\hline Prostate volume in ml, median (IQR) & $46(32-62)$ & $44(31-58)$ & $54(39-70)$ & $<0.001$ \\
\hline
\end{tabular}


Table 1 Patient and biopsy characteristics (Continued)

\begin{tabular}{|c|c|c|c|c|}
\hline & Total & 2D TRUS & 3D TRUS & $P$ value \\
\hline \multicolumn{5}{|l|}{ Type of operator: } \\
\hline - Experienced, n (\%) & $200(52.6 \%)$ & $130(45.5 \%)$ & $70(74.5 \%)$ & $<0.001$ \\
\hline - Less experienced, n (\%) & $180(47.4 \%)$ & $156(54.5 \%)$ & $24(25.5 \%)$ & \\
\hline Biopsy cores per session, mean (SD) & 12.6 (SD 1.56) & 12.7 (SD 1.53) & 12.6 (SD 1.67) & 0.519 \\
\hline Biopsy sessions with fragmentation, $\mathrm{n}(\%)$ & $113(29.7 \%)$ & $113(39.2 \%)$ & 0 & $<0.001$ \\
\hline Length of biopsy cores in mm, mean (SD) ${ }^{a}$ & 13.81 (SD: 3.05) & 12.54 (SD: 2.96) & 16.10 (SD: 1.48) & $<0.001$ \\
\hline
\end{tabular}

aiopsy sessions with fragmentation were excluded for these analyses

prostate volume and type of operator included as covariates, the 3D TRUS cohort remained significantly associated with detection of PCa (aOR 1.33, 95\% CI 1.03-1.72) and clinically significant PCa (aOR 1.47, 95\% CI 1.091.98). The association between 3D TRUS and detection of (clinically significant) PCa on multivariate regression analysis for the subgroups demonstrated significance for clinically significant $\mathrm{PCa}$ in the biopsy naive group (aOR $1.41,95 \% \mathrm{CI} 1.02-1.96)$ and for $\mathrm{PCa}$ in the prior negative group (aOR 1.78, 95\% CI (1.04-3.04) (Table 2).

\section{PCa positive biopsies: biopsy characteristics and Gleason score}

Tumour biopsy characteristics between the 3D GS TRUS cohort and the 2D GS TRUS cohort in patients with positive PCa biopsies were not statistically different as the amount of positive biopsy cores, the ratio positive biopsy cores out of the total biopsy cores and the percentage of tumour volume per PCa biopsy session did not differ between both groups (Additional file 3). The biopsy Gleason score among patients with $\mathrm{PCa}$ positive biopsies was significantly different between the 3D TRUS and 2D TRUS cohort in the whole group and biopsy naive subgroup analyses as more clinically significant and Gleason score $\geq 7 \mathrm{PCa}$ was found in the 3D TRUS cohort $(P<0.05)$ (Additional file 4 ).

\section{Discussion}

Limited studies with small sample sizes have been performed to assess the value of 3D GS TRUS in guiding prostate biopsies. Peltier et al. published the first cohort study into 3D TRUS-guided prostate biopsy and detection rate of $\mathrm{PCa}$. In 220 consecutive biopsy naive men, detection rate of $\mathrm{PCa}$ was significantly higher in the 3D TRUS group using the Urostation (Koelis ${ }^{\circ}$ ) compared with 2D TRUS $(50.0 \%$ versus $33.6 \%, p<0.05)$ [17]. In contrary, a previous study carried out in our institution with 325 men showed no added value of 3D TRUS guidance compared to $2 \mathrm{D}$ with all biopsy cores preembedded (aOR 0.79, 95\% CI 0.47-1.34) [25].

This present study, covering a period of 9 full years with 1618 prostate biopsy sessions, demonstrated higher detection rates of $\mathrm{PCa}$ and clinically significant $\mathrm{PCa}$ with the use of 3D TRUS-guidance and preembedding compared to conventional 2D GS TRUS-guidance. These findings may be explained by different hypotheses. On one side, 3D TRUS could have improved detection as it offers the ability to accurately register the location of the biopsy needle tract within the gland, possibly leading to better distribution of biopsy cores; instead needle placement with 2D-guidance is not always homogeneously distributed and tends to cluster.[15, 26-28] Besides, length of biopsy cores was significantly larger and fragmentation occurred significantly less in the 3D GS TRUS cohort possibly also aiding detection of $\mathrm{PCa}$ as Iczkowski et al. and Rogatsch et al. demonstrated in their studies that length of biopsy tissue significantly correlates with $\mathrm{PCa}$ detection rate and higher frequency of PCa diagnosis was found with an improved preembedding method, respectively $[19,20,25]$.

Multiparametric MRI (mpMRI) of the prostate is increasingly used in the diagnostic pathway of PCa and three large studies, evaluating the detection rates of an MRI-targeted biopsy approach and TRUS guided systematic biopsy approach, have recently been performed in biopsy-naïve men [30-32].

While the PRECISION trial demonstrated that an MRI targeted biopsy approach detected significantly more clinically significant $\mathrm{PCa}$ in comparison with a TRUS systematic biopsy approach both the MRI First and $4 \mathrm{M}$ Study demonstrated comparable detection rates of clinically significant PCa between the standard TRUS systematic biopsy and MRI targeted biopsy approach.

Obtaining an mpMRI before biopsy improves the detection of clinically significant PCa but at present does not avoid the need for systematic biopsy as shown in the systematic review from Moldovan et al. and the MRI FIRST study where 10 to $15 \%$ of clinically significant PCa were still missed in men with a negative mpMRI [31,33]. Even in the Dutch $4 \mathrm{M}$ study with high-quality MRI standards, $7 \%(21 / 317)$ of all men with a suspicious mpMRI scan had clinically significant PCa only on systematic biopsy [32]. A high-quality TRUS systematic biopsy, possibly with the use of 3D TRUS-guidance and preembedding, could therefore still be important in the current diagnostic setting where mpMRI is also included. 
Table 2 Detection rate of all prostate cancer and clinically significant prostate cancer

\begin{tabular}{|c|c|c|c|}
\hline \multicolumn{4}{|l|}{ Whole group } \\
\hline \multicolumn{4}{|c|}{ Detection rate of $\mathrm{PCa}$} \\
\hline Biopsy cohort: & $\mathrm{n} / \mathrm{N}(\%)$ & Crude OR $(95 \% \mathrm{Cl})$ & $\mathrm{aOR}^{\mathrm{a}}(95 \% \mathrm{Cl})$ \\
\hline Total & $568 / 1618(35.1 \%)$ & & \\
\hline 3D TRUS & 183/469 (39.0\%) & $1.27(1.02-1.59)$ & $1.33(1.03-1.72)$ \\
\hline 2D TRUS & $385 / 1149(33.5 \%)$ & Reference & Reference \\
\hline \multicolumn{4}{|c|}{ Detection rate of clinically significant $P C a$} \\
\hline & $\mathrm{n} / \mathrm{N}(\%)$ & Crude OR (95\% Cl) & $\mathrm{aOR}^{\mathrm{a}}(95 \% \mathrm{Cl})$ \\
\hline Total & $335 / 1618(20.7 \%)$ & & \\
\hline 3D TRUS & $117 / 469(24.9 \%)$ & $1.42(1.10-1.83)$ & $1.47(1.09-1.98)$ \\
\hline 2D TRUS & $218 / 1149(19.0 \%)$ & Reference & Reference \\
\hline \multicolumn{4}{|l|}{ Biopsy naive group } \\
\hline \multicolumn{4}{|c|}{ Detection rate of $P C a$} \\
\hline & $\mathrm{n} / \mathrm{N}(\%)$ & Crude OR $(95 \% \mathrm{Cl})$ & $\mathrm{aOR}^{\mathrm{b}}(95 \% \mathrm{Cl})$ \\
\hline Total & $466 / 1238(37.6 \%)$ & & \\
\hline 3D TRUS & 151/375 (40.3\%) & $1.17(0.92-1.50)$ & $1.20(0.90-1.60)$ \\
\hline 2D TRUS & $315 / 863(36.5 \%)$ & Reference & Reference \\
\hline \multicolumn{4}{|c|}{ Detection rate of clinically significant $P C a$} \\
\hline & $\mathrm{n} / \mathrm{N}(\%)$ & Crude OR $(95 \% \mathrm{Cl})$ & $\mathrm{aOR}^{\mathrm{b}}(95 \% \mathrm{Cl})$ \\
\hline Total & $295 / 1238(23.8 \%)$ & & \\
\hline 3D TRUS & 105/375 (28.0\%) & $1.38(1.04-1.82)$ & $1.41(1.02-1.96)$ \\
\hline 2D TRUS & 190/863 (22.0\%) & Reference & Reference \\
\hline \multicolumn{4}{|c|}{ Prior negative group } \\
\hline \multicolumn{4}{|c|}{ Detection rate of $P C a$} \\
\hline & $\mathrm{n} / \mathrm{N}(\%)$ & Crude OR $(95 \% \mathrm{Cl})$ & $\mathrm{aOR}^{\mathrm{C}}(95 \% \mathrm{Cl})$ \\
\hline Total & $102 / 380(26.8 \%)$ & & \\
\hline 3D TRUS & 32/94 (34.0\%) & $1.59(0.96-2.64)$ & $1.78(1.04-3.04)$ \\
\hline 2D TRUS & $70 / 286(24.5 \%)$ & Reference & Reference \\
\hline \multicolumn{4}{|c|}{ Detection rate of clinically significant $P C a$} \\
\hline & $\mathrm{n} / \mathrm{N}(\%)$ & Crude OR $(95 \% \mathrm{Cl})$ & $\mathrm{aOR}^{\mathrm{d}}(95 \% \mathrm{Cl})$ \\
\hline Total & $40 / 380(10.5 \%)$ & & \\
\hline 3D TRUS & 12/94 (12.8\%) & $1.35(0.66-2.77)$ & - \\
\hline 2D TRUS & $28 / 286(9.8 \%)$ & Reference & Reference \\
\hline
\end{tabular}

adjusted Odds Ratio: adjusted for age, DRE, prostate volume and physician (PSA and Type of biopsy session resulted in $<10 \%$ change in $\beta$ ) ${ }^{b}$ adjusted Odds Ratio: adjusted for age, DRE and prostate volume and physician (PSA resulted in $<10 \%$ change in the $\beta$ )

' adjusted Odds Ratio: adjusted for age and Log (prostate volume), (age, PSA, DRE and physician resulted in $<10 \%$ change in the $\beta$ )

${ }^{d}$ the number of events per variable are too low to be analyzed in multivariable logistic regression analysis

On the other side, as a retrospective study, our analysis has important limitations that could have influenced the outcome. First of all, biopsy sessions after September 2013 were performed with 3D TRUS guidance and the preembedding method; as a consequence, their independent influence on the detection rate of $\mathrm{PCa}$ cannot be assessed. However as both procedures act on different aspects of $\mathrm{PCa}$ diagnosis, a joint positive effect was hypothesized over masking of independent results. Secondly, difference in study time period could have had an impact on PCa detection rate as different unidentified factors influencing $\mathrm{PCa}$ detection rate could have changed during these time periods. Prostate biopsy selection in both cohorts however was not based on important pre-biopsy tests such as mpMRI and/or novel biomarkers. Both cohorts differed in baseline characteristics with older age, lower prostate volume and a biopsy naive setting all associated with higher odds of $\mathrm{PCa}$ and significant $\mathrm{PCa}$ on univariate and multivariate analysis in our study (Additional file 1: Table S1). Beside this, we decided in our study to include the Large Grade Group 1 (GS $3+3)$ as clinically significant PCa. Recent EAU guidelines use the definition of $G G \geq 2(G S \geq 3+4)$ for clinical significant PCa. This matches with the newly introduced ISUP scoring system, where no separation is made between large and small GG 1 (GS $3+3) \mathrm{PCa}$ [29]. We decided to include large GS $3+3=6 \mathrm{PCa}$ as clinically significant as this is currently still used as a criteria for active surveillance versus radical treatment in low-risk PCa patients.

In addition, experience level of operators was various. Although a correction strategy was implemented, residual confounding could still be possible. Last, $32.4 \%$ (944/2917) of all our prostate biopsy sessions were excluded on the basis of PSA inclusion criteria. In line with the literature we excluded men with high PSA levels as improved detection of significant PCa cancer is most necessary in the PSA grayzone from 4 to $10 \mathrm{ng} / \mathrm{mL}$, while men with higher PSA levels tend to be diagnosed accurately with TRUS-guided systematic biopsies [30-32].

As a result, association between 3D TRUS-guidance with preembedding and detection of PCa could be partially biased on the basis of confounding and selection.

Despite these limitations in relation to the detection of $\mathrm{PCa}$, there are other possible clinical applications for our approach. The 3D TRUS system is not only developed for direct imaging and navigation, but also for procedure planning with recording and integration of exact locations of biopsy cores and updating of pathology results. With the possibility to assess the exact spatial coordinates of each previous needle trajectory, a 'biopsy map' can be built based on (positive) previous core taken and adequate sample sites can potentially be chosen in prior negative patients or in patients in active surveillance. Additionally, in our study biopsy core length and Gleason score were significantly higher in the cohort with 3D GS TRUS-guidance and preembedding. As more biopsy core length from a presumably spatial better biopsied prostate gland can be pathologically examined, correlation with prostatectomy specimens could possibly be improved and thereby prevent men with unfavourable disease to be placed in active surveillance or less 
aggressive treatments. However caution should be exercised for interpretation of Gleason score differences between both cohorts as Gleason grading of PCa was updated at the end of 2014 during an ISUP Consensus Conference [29].

As a consequence, higher Gleason scores in the 3D TRUS cohort could be partially the result of these new grading pattern definitions. We will therefore investigate these potential applications in future prospective studies and pathology concordance studies. These findings highlight the need for future research regarding the complementary value of 3D TRUS guidance and preembedding in the combination with mpMRI and other new diagnostic applications.

\section{Conclusion}

The current study suggests an added value of 3D TRUS-guidance and preembedding compared to conventional 2D GS TRUS-guidance regarding detection rate of $\mathrm{PCa}$ and clinically significant $\mathrm{PCa}$ among patients undergoing prostate biopsies. At the same time, 3D TRUS-guidance and preembedding provided significant more biopsy core tissue length and higher biopsy Gleason scores. Additional prospective studies and studies concerning pathology correlation are needed to justify the routine application of 3D GS TRUS-guidance and biopsy core preembedding.

\section{Additional files}

Additional file 1: Table S1. Univariate and multivariate logistic regression analyses of individual risk factors for detection of prostate cancer and clinically significant prostate cancer. (DOCX $14 \mathrm{~kb}$ )

Additional file 2: SmartB ${ }^{\mathrm{TM}}$ device prostate biopsy core preembedding. (PPTX $95 \mathrm{~kb}$ )

Additional file 3: Tumour biopsy characteristics among patients with positive biopsies. (XLSX $12 \mathrm{~kb}$ )

Additional file 4: Biopsy Gleason score among patients with positive biopsies. (XLSX 13 kb)

\section{Abbreviations}

2D: Two-dimensional; 3D: Three-dimensional; Cl: Confidence interval; DRE: Digital rectal examination; GS: Greyscale; IQR: Interquartile range; mpMRI: Multiparametric Magnetic Resonance Imaging; OR: Odds ratio; PCa: Prostate cancer; PSA: prostate-specific antigen; SD: Standard deviation; TRUS: Transrectal ultrasound

\section{Acknowledgements}

Not applicable.

Funding

No funding.

\section{Availability of data and materials}

All data generated or analysed during this study are included in this published article and its supplementary information files.

\section{Authors' contributions}

AvdA conceived the idea for the study, made the protocol and wrote the manuscript with CKM. CKM performed the data collection, conducted the analyses and wrote the manuscript with AvdA. MG made the protocol and assisted with the data collection. AvdA, CKM and MG had access to all of the research data. JvdL revised necessary pathology. JvdL, HB, BS, MM and HW assisted with protocol development. HB, BS, MS, MM and HW contributed to the interpretation of the data and critically revised earlier draft versions. All. authors read and approved the final manuscript.

\section{Ethics approval and consent to participate}

The ethical committee of the Jeroen Bosch Hospital gave ethical approval for this study with a non-WMO declaration. Informed consent for the current study was not required, because the data-collection was retrospective in design.

\section{Consent for publication}

Not applicable.

\section{Competing interests}

All authors declares that they have no conflict of interests.

\section{Publisher's Note}

Springer Nature remains neutral with regard to jurisdictional claims in published maps and institutional affiliations.

\section{Author details}

'Department of Urology, Jeroen Bosch Hospital, Post office box 90153, 's-Hertogenbosch 5200 ME, The Netherlands. '2Department of Pathology, Jeroen Bosch Hospital, 's-Hertogenbosch, The Netherlands. ${ }^{3}$ Department of Urology, Radboud University Medical Centre, Nijmegen, The Netherlands.

${ }^{4}$ Electrical Engineering, Eindhoven University of Technology, Eindhoven, The Netherlands. ${ }^{5}$ Department of Urology, AMC University Hospital, Amsterdam, The Netherlands.

Received: 6 August 2018 Accepted: 31 March 2019

Published online: 16 April 2019

\section{References}

1. Arnold M, Karim-Kos HE, Coebergh JW, Byrnes G, Antilla A, Ferlay J, et al. Recent trends in incidence of five common cancers in 26 European countries since 1988: analysis of the European Cancer observatory. Eur J Cancer. 2015;51(9):1164-87.

2. Schröder FH, Hugosson J, Carlsson S, Tammela TL, Määttänen L, Auvinen A, et al. Screening for prostate Cancer decreases the risk of developing metastatic disease: findings from the European randomized study of screening for prostate Cancer. Eur Urol. 2012;62(5):745-52.

3. Schröder FH, Hugosson J, Roobol MJ, Tammela TL, Zappa M, Nelen V, et al. Screening and prostate cancer mortality: results of the European randomised study of screening for prostate Cancer (ERSPC) at 13 years of follow-up. Lancet. 2014:384(9959):2027-35.

4. Wolf AM, Wender RC, Etzioni RB, Thompson IM, D'Amico AV, Volk RJ, et al. American Cancer Society guideline for the early detection of prostate cancer: update 2010. CA Cancer J Clin. 2010;60(2):70-98.

5. Smeenge $\mathrm{M}$, de la Rosette JJ, Wijkstra $\mathrm{H}$. Current status of transrectal ultrasound techniques in prostate cancer. Curr Opin Urol. 2012;22(4): 297-302.

6. Ukimura O, Coleman JA, de la Taille A, Emberton M, Epstein II, Freedland SJ, et al. Contemporary role of systematic prostate biopsies: indications, techniques and implications for patient care. Eur Urol. 2013;63(2):214-30.

7. Wilkinson BA, Hamdy FC. State-of-the-art staging in prostate cancer. BJU Int 2001:87(5):423-31.

8. Ploussard G, Salomon L, Xylinas E, Allory Y, Vordos D, Hoznek A, et al. Pathological findings and prostate specific antigen outcomes after radical prostatectomy in men eligible for active surveillance--does the risk of misclassification vary according to biopsy criteria? J Urol. 2010; 183(2):539-44.

9. Bul M, Zhu X, Rannikko A, Staerman F, Valdagni R, Pickles T, et al. Radical prostatectomy for low-risk prostate cancer following initial active surveillance: results from a prospective observational study. Eur Urol. 2012 62(2):195-200

10. Bjurlin MA, Carter HB, Schellhammer P, Cookson MS, Gomella LG, Troyer D, et al. Optimization of initial prostate biopsy in clinical practice: sampling, labeling and specimen processing. J Urol. 2013;189(6):2039-46. 
11. Sedelaar JP, van Roermund JG, van Leenders GL, van de Kaa CA H, Wijkstra $\mathrm{H}$, de la Rosette JJ. Three-dimensional grayscale ultrasound: evaluation of prostate cancer compared with benign prostatic hyperplasia. Urology. 2001; 57(5):914-20.

12. Mehta SS, Azzouzi AR, Hamdy FC. Three dimensional ultrasound and prostate cancer. World J Urol. 2004 Nov;22(5):339-45.

13. Mitterberger M, Pinggera GM, Pallwein L, Gradl J, Frauscher F, Bartsch G, et al. The value of three-dimensional transrectal ultrasonography in staging prostate cancer. BJU Int. 2007 Jul;100(1):47-50

14. Cool D, Sherebrin S, Izawa J, Chin J, Fenster A. Design and evaluation of a 3D transrectal ultrasound prostate biopsy system. Medical physics. 2008; 35(10):4695-707.

15. Cohen M, Cytron S, Nativ O, Shmitz A, Yudkevich B, Shental J. 816 3D TRUS prostate biopsy recording and guidance - the Navigo system. Eur Urol Suppl. 2012;11(1):e816,e816a.

16. Chin JL, Downey DB, Mulligan M, Fenster A. Three-dimensional transrectal ultrasound guided cryoablation for localized prostate cancer in nonsurgical candidates: a feasibility study and report of early results. J Urol. 1998;159(3): 910-4.

17. Peltier A, Aoun F, El-Khoury F, Hawaux E, Limani K, Narahari K, et al. 3D versus 2D systematic Transrectal ultrasound-guided prostate biopsy: higher Cancer detection rate in clinical practice. Prostate cancer. 2013; 2013:783243.

18. Pryaluhkin AE, Vandromme A, Dellmann A, Donhuijsen K, Hammerer PG. Prostate biopsy core handling: comparison of contemporary preembedding methods. Urol Int. 2015;95(2):203-8.

19. Rogatsch H, Mairinger T, Horninger W, Gschwendtner A, Bartsch G, Mikuz G. Optimized preembedding method improves the histologic yield of prostate core needle biopsies. Prostate. 2000;42(2):124-9.

20. Rogatsch H, Moser P, Volgger H, Horninger W, Bartsch G, Mikuz G, et al. Diagnostic effect of an improved preembedding method of prostate needle biopsy specimens. Hum Pathol. 2000;31(9):1102-7.

21. Cohen M, Shefler A, Patsiansky S, Shapira-Schweitzer K, Nativ O, Bejar J. The SmartBX ${ }^{\mathrm{TM}}$ - initial experience with a semi-automated prostate biopsy core download system. Poster presented at the 6th international symposium on focal therapy and imaging in prostate and kidney Cancer, Amsterdam. Poster retrieved from: https://www.focaltherapy.org/2013/Poster_Abstracts/ Focal_2013_pP-22a.pdf.

22. Bokhorst LP, Zhu X, Bul M, Bangma CH, Schröder FH, Roobol MJ. Positive predictive value of prostate biopsy indicated by prostate-specific-antigenbased prostate cancer screening: trends over time in a European randomized trial*. BJU Int. 2012;110(11):1654-60.

23. Roobol MJ, van Vugt HA, Loeb S, Zhu X, Bul M, Bangma CH, et al. Prediction of prostate cancer risk: the role of prostate volume and digital rectal examination in the ERSPC risk calculators. Eur Urol. 2012;61(3):577-83. https://doi.org/10.1016/j.eururo.2011.11.012

24. Lawrentschuk N, Toi A, Lockwood GA, Evans A, Finelli A, O'Malley M, et al. Operator is an independent predictor of detecting prostate cancer at transrectal ultrasound guided prostate biopsy. J Urol. 2009;182(6):2659-63.

25. Gayet M, van der Aa A, Schmitz P, Beerlage HP, Schrier BP, Mulders PF, et al. 3D Navigo ${ }^{\mathrm{TM}}$ versus TRUS-guided prostate biopsy in prostate cancer detection. World J Urol. 2016;34(9):1255-60.

26. Cool DW, Connolly MJ, Sherebrin S, Eagleson R, Izawa JI, Amann J, et al. Repeat prostate biopsy accuracy: simulator-based comparison of two- and three dimensional transrectal USmodalities. Radiology. 2010;254(2):587-94.

27. Wan G, Wei Z, Gardi L, Downey DB, Fenster A. Brachytherapy needle deflection evaluation and correction. Med Phys. 2005;32:902-9.

28. Iczkowski KA, Casella G, Seppala RJ, Jones GL, Mishler BA, Qian J, et al. Needle core length in sextant biopsy influences prostate cancer detection rate. Urology. 2002;59(5):698-703.

29. Epstein Jl, Egevad L, Amin MB, Delahunt B, Srigley JR, Humphrey PA, et al. The 2014 International Society of Urological Pathology (ISUP) consensus conference on Gleason grading of prostatic carcinoma: definition of grading patterns and proposal for a new grading system. Am J Surg Pathol. 2016;40(2):244-52.

30. Kasivisvanathan V, Rannikko AS, Borghi M, Panebianco V, Mynderse LA, Vaarala $\mathrm{MH}$, et al. MRI-targeted or standard biopsy for prostate-Cancer diagnosis. N Engl J Med. 2018;378(19):1767-77. https://doi.org/10.1056/ NEJMoa1801993

31. Rouvière $O$, Puech $P$, Renard-Penna $R$, Claudon $M$, Roy $C$, Mège-Lechevallier F. Use of prostate systematic and targeted biopsy on the basis of multiparametric MRI in biopsy-naive patients (MRI-FIRST): a prospective, multicentre, paired diagnostic study. Lancet Oncol. 2019;20(1):100-9.

32. van der Leest M, Cornel E, Israel B, Hendriks R, Padhani AR, Hoogenboom M, et al. Head-to-head comparison of Transrectal ultrasound-guided prostate biopsy versus multiparametric prostate resonance imaging with subsequent magnetic resonance-guided biopsy in biopsy-naïve men with elevated prostate-specific antigen: a large prospective multicenter clinical study. Eur Urol. 2018; In Press.

33. Moldovan PC, van den Broeck T, Sylvester R, Marconi L, Bellmunt J, van den Bergh RCN, et al. What is the negative predictive value of multiparametric magnetic resonance imaging in excluding prostate Cancer at biopsy? A systematic review and meta-analysis from the European Association of Urology prostate Cancer guidelines panel. Eur Urol. 2017;72(2):250-66.

\section{Ready to submit your research? Choose BMC and benefit from:}

- fast, convenient online submission

- thorough peer review by experienced researchers in your field

- rapid publication on acceptance

- support for research data, including large and complex data types

- gold Open Access which fosters wider collaboration and increased citations

- maximum visibility for your research: over $100 \mathrm{M}$ website views per year

At BMC, research is always in progress.

Learn more biomedcentral.com/submissions 